\title{
Formation of Mn-Related Defect Band in $\operatorname{InP}$
}

\author{
B. MACIEJA ${ }^{a, *}$, K.P. Korona ${ }^{a}$, M. Piersa ${ }^{b}$, A.M. Witowski $^{a}$,

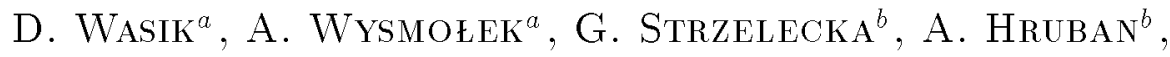 \\ B. Surma ${ }^{b}$, M. PalczeWska ${ }^{b}$, M. Kamińska ${ }^{a}$ \\ AND A. TWARDOWSKI ${ }^{a}$ \\ ${ }^{a}$ Institute of Experimental Physics, Warsaw University \\ Hoża 69, 00-681 Warsaw, Poland \\ ${ }^{b}$ Institute of Electronic Materials Technology \\ Wólczyńska 133, 01-919 Warsaw, Poland
}

\begin{abstract}
Electron paramagnetic resonance, optical absorption, luminescence and electrical studies of InP highly doped with Mn were performed. Electron paramagnetic resonance revealed presence of manganese in $\mathrm{Mn}^{2+}\left(d^{5}\right)$ configuration. In optical absorption, systematic reduction of InP band gap was observed with increase in Mn content. This was correlated with increase in photoionization-type absorption band starting at $0.2 \mathrm{eV}$. Time-resolved photoluminescence measurements showed decrease in photoexcited carrier lifetime and shortening of donor-acceptor pair recombination time with increase in $\mathrm{Mn}$ content. Moreover, photoluminescence band was shifted to lower energies, similarly to optical band gap. In electrical transport two mechanisms of conductivity were observed. Valence band transport dominated at higher temperatures, above $160 \mathrm{~K}$, and activation energy of free-hole concentration was determined as about $0.20 \mathrm{eV}$. At lower temperatures hopping conductivity, clearly related to $\mathrm{Mn}$ defect band, was present. All these results were consistent with assumption of creation of Mn-related defect-band at $0.2 \mathrm{eV}$ above InP valence band. It was found that $\mathrm{Mn}$ centers responsible for this band were in configurations of either $d^{5}$ or $d^{5}$ plus a hole localized about $7 \AA$ around corresponding Mn core.
\end{abstract}

PACS numbers: 71.55.Eq, 72.20.Ee, 72.20.Jv

\section{Introduction}

Recent theoretical predictions of possible ferromagnetism at room temperature in some of diluted magnetic III-V compounds trigerred many studies of such

*corresponding author; e-mail: barmac@mg.gov.pl 
materials. Up to now the record high Curie temperature, about $150 \mathrm{~K}$ [1], belongs to GaMnAs. However, in spite of huge effort put in optimization of growth parameters and post-growth annealing, this temperature is still far below $300 \mathrm{~K}$, convenient temperature for application purposes. Therefore, it seems important to study diluted magnetic semiconductors based on other III-V compounds in order to search for possible better materials. The important issue seems to be the understanding of mechanisms leading to ferromagnetism in Mn-based III-V compounds, and especially conditions for Mn centers necessary to fulfill in order to obtain high temperature ferromagnetic ordering.

In this paper we report results of our studies performed on InP highly doped with Mn. InP was grown by Czochralski method. Manganese was added during growth and its concentration reached $8 \times 10^{18} \mathrm{~cm}^{-3}$ value. Because of segregation effect, it was possible to select samples from the same ingot with different Mn concentration (samples Mn I, Mn II, and Mn III). Glow discharge mass spectroscopy (GDMS) showed that Mn content was $5 \times 10^{18} \mathrm{~cm}^{-3}, 7 \times 10^{18} \mathrm{~cm}^{-3}$, and $8 \times 10^{18} \mathrm{~cm}^{-3}$ in samples Mn I, Mn II, and Mn III, respectively. Concentration of other acceptor impurities (mainly $\mathrm{Zn}$ ) was about $10^{16} \mathrm{~cm}^{-3}$. Concentration of donor impurities (mainly Si) was about $5 \times 10^{17} \mathrm{~cm}^{-3}$. Two Czochralski-grown samples without manganese: InP:S ( $n$-type) and InP:Zn (Hall concentration $p=$ $5 \times 10^{17} \mathrm{~cm}^{-3}$ at $T=300 \mathrm{~K}$ ) were also studied for comparison.

\section{Experimental results}

Electron paramagnetic resonance (EPR) studies of the InP samples described above were performed using Bruker spectrometer working at about $9 \mathrm{GHz}$ microwave frequency. They revealed presence of manganese in $\mathrm{Mn}^{2+}\left(d^{5}\right)$ configuration for all Mn doped InP. The corresponding spectra are presented in Fig. 1. They show characteristic signal with $g$ factor equal to 2.00 , similar to the one already reported by others [2]. Broadening of the line is due to interaction of Mn centers in highly doped samples.

Continuous-excitation (CW) photoluminescence (PL) was measured, using He-Ne laser $(633 \mathrm{~nm})$ or second harmonic of Nd:YAG laser $(531 \mathrm{~nm})$ for excitation and $50 \mathrm{~cm}$ monochromator equipped with germanium detector for analysis of PL spectra. In time-resolved photoluminescence (TRPL) measurements, $150 \mathrm{fs}$ pulses of Ti:sapphire laser were used for photoexcitation of free electron-hole pairs in the samples. Then a monochromator and streak camera analyzed the spectral and temporal distribution of the PL. The photoluminescence spectra of InP:Zn revealed excitonic luminescence lines and shallow donor-shallow acceptor recombination. An acceptor bound line $\mathrm{A}^{0} \mathrm{X}$ at $1.413 \mathrm{eV}(T=8 \mathrm{~K})$ and lifetime $70 \pm 10 \mathrm{ps}$ was observed. For InP:Mn samples, strong decrease in PL intensity in comparison with non Mn-doped samples was observed. Excitonic lines were hardly visible. In the energy range of $1.3-1.4 \mathrm{eV}$ PL mostly weak spectrum, corresponding to 


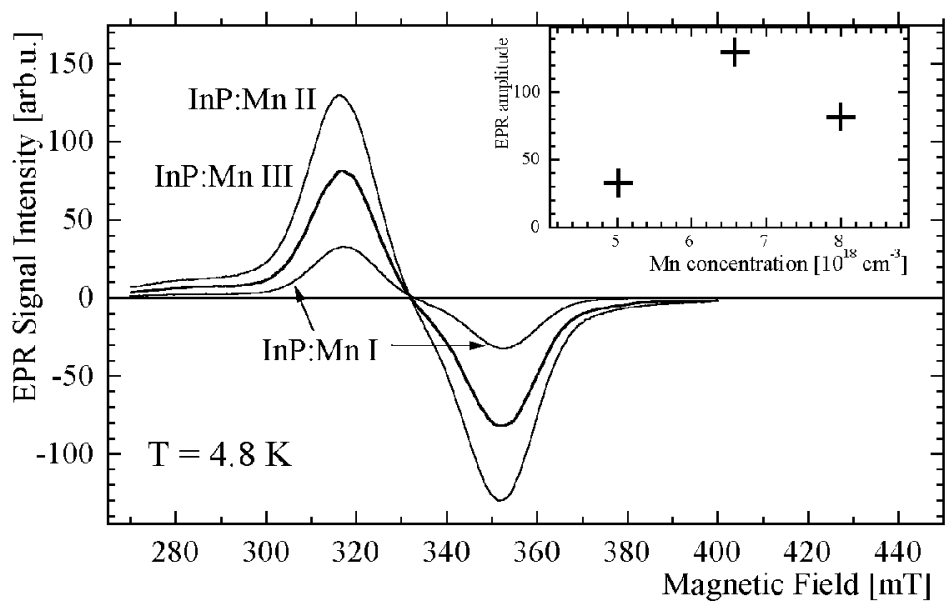

Fig. 1. Electron paramagnetic resonance spectrum for InP:Mn samples. The observed line is due to $\mathrm{Mn}^{2+}\left(d^{5}\right)$ configuration $(g=2.00)$. Inset: the EPR amplitude vs. Mn content dependence. The dependence is weak since the signal comes from minor $\mathrm{Mn}^{2+}$ state.

shallow donor to shallow acceptor ( $\mathrm{D}-\mathrm{A})$ transitions peaked at $1.355 \mathrm{eV}(T=8 \mathrm{~K})$, was observed. Broad luminescence band in the energy range at about $0.9-1.2 \mathrm{eV}$ dominated the PL spectrum. This band could be assigned to shallow donor-Mn acceptor (D-Mn) recombination, as it was shifted about $0.2 \mathrm{eV}$ below the excitonic transitions.

Estimation from TRPL results gave photoexcited carriers lifetime in InP:Mn of the order of a few tens of femtoseconds. Taking into account that recombination rate $r_{S}$ is proportional to the concentration of Mn centers $N_{\mathrm{Mn}}, r_{S}=R_{\mathrm{Mn}} N_{\mathrm{Mn}}$, one could estimate recombination coefficient $R_{\mathrm{Mn}}=10^{-5} \mathrm{~cm}^{3} / \mathrm{s}$. The increase in $\mathrm{Mn}$ concentration in $\mathrm{InP}$ samples leads to decrease in lifetime of the $\mathrm{D}-\mathrm{A}$ line. The decay time observed for InP:Zn samples was $\tau_{\mathrm{D}}=430 \mathrm{ps}$. It was reduced to $\tau_{\mathrm{D}}=200 \pm 50 \mathrm{ps}, \tau_{\mathrm{D}}=150 \pm 30 \mathrm{ps}, \tau_{\mathrm{D}}=75 \pm 10 \mathrm{ps}$ for samples MnI, MnII, and MnIII, respectively. Therefore it was clear that $\mathrm{Mn}$ content leads to faster decay of $\mathrm{D}-\mathrm{A}$ luminescence.

Optical absorption was measured in the wide range of energy, from $40 \mathrm{meV}$ to $1.5 \mathrm{eV}$, using CARY5 and Bruker $113 \mathrm{~V}$ spectrometers. In energy region close to InP band-to-band transitions, systematic reduction of band gap was observed with increase in Mn content. We present this behavior in Fig. 2, together with results of the measurements performed on InP samples with no intentional Mn doping. At lower energies absorption band appeared for Mn-doped samples with a threshold at about $0.2 \mathrm{eV}$ - see Fig. 3. This band has been already observed by Lambert et al. [3] and assigned to ionization of hole from Mn center to InP valence band. However, the absorption band observed by us is broader and structureless. This indicates, together with broadening of band-to-band transitions, creation of 


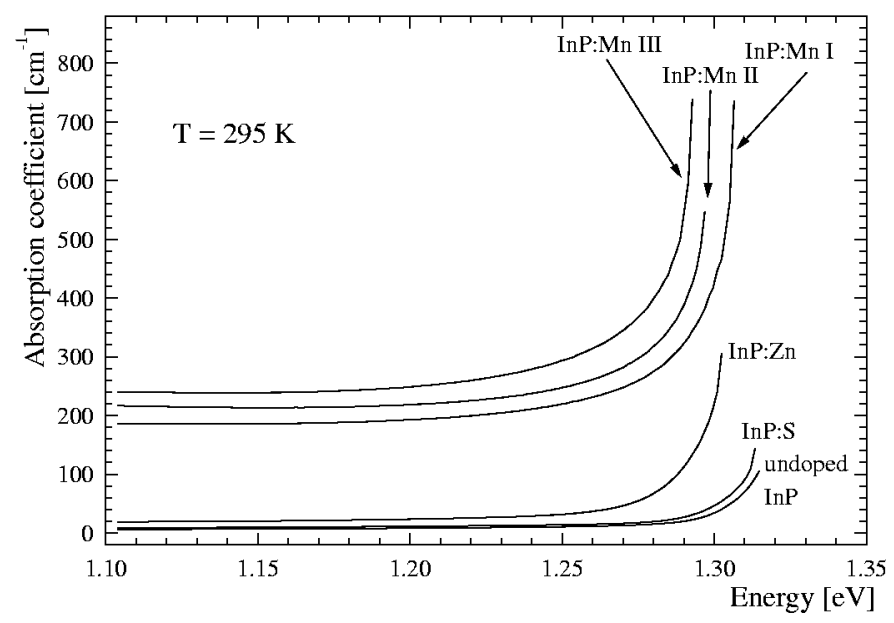

Fig. 2. Optical absorption spectrum in the range of InP band-to-band transitions for InP:Mn (samples MnI, MnII, and MnIII), for InP:S and for InP:Zn. Reduction of band gap for InP:Mn is clearly visible.

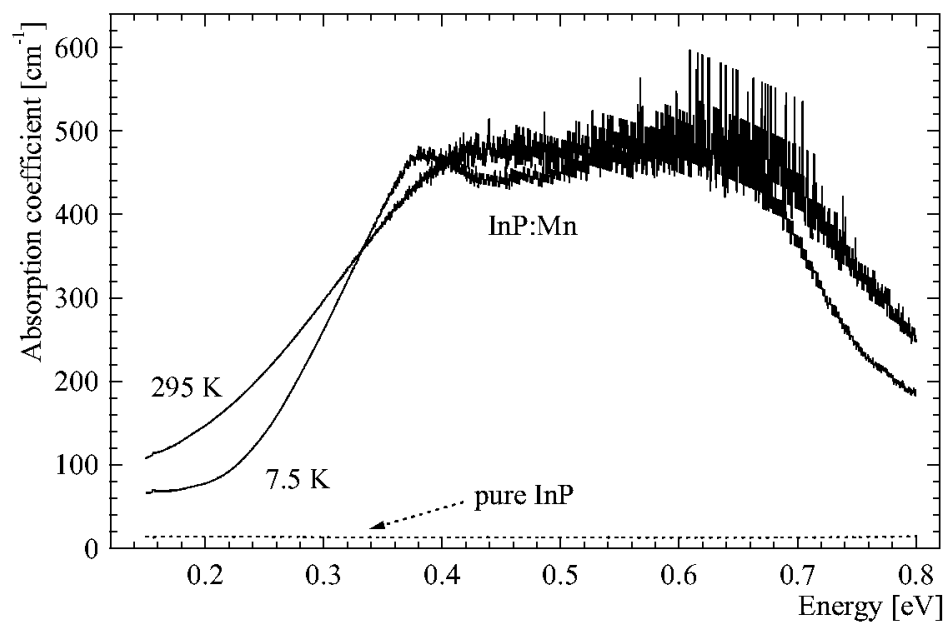

Fig. 3. Photoionization spectrum of $\mathrm{Mn}$ in $\mathrm{InP}$ corresponding to hole transition from $\mathrm{Mn}$ level to InP valence band. For comparison absorption of InP:S in the same energy range is shown.

Mn-related impurity band. This band is placed about $0.2 \mathrm{eV}$ above $\mathrm{InP}$ valence band. Further experiments were performed in order to support this thesis as well as to characterize Mn center responsible for the impurity band.

Hall effect measurements were performed on the three InP:Mn samples with different $\mathrm{Mn}$ content in the temperature range of 60-300 K. Temperature-dependent electrical transport revealed two mechanisms of conductivity. At high tempera- 
tures, above $160 \mathrm{~K}$, valence band transport dominated, whereas at low temperatures hopping conductivity was present. In the case of hopping conductivity, the conductivity is given by the formula

$$
\sigma_{\text {Hо }}=\sigma_{\mathrm{H} \circ \mathrm{O}} \exp \left(-1.73 N^{-1 / 3} / a_{f}-E_{3} / k T\right),
$$

where $N$ is concentration of defects, which pins Fermi level, and $a_{f}$ has a meaning of localization radius of a carrier (in our case hole) trapped by the defect [4]. The activation energy $E_{3}$ describes potential fluctuations in the hopping band. In the case of two transport mechanisms, the effective conductivity tensor $\hat{\sigma}_{\text {ef }}$ is a sum of two tensors: $\hat{\sigma}_{\mathrm{ef}}=\hat{\sigma}_{\mathrm{VB}}+\hat{\sigma}_{\mathrm{Ho}}$. Therefore, the effective mobility $\mu_{\mathrm{ef}}$ measured in presence of the both transport mechanisms is $\mu_{\mathrm{ef}}=\mu_{\mathrm{VB}} \sigma_{\mathrm{VB}} /\left(\sigma_{\mathrm{VB}}+\sigma_{\mathrm{Ho}}\right)$ and the effective hole concentration is $p_{\mathrm{ef}}=\left(\sigma_{\mathrm{VB}}+\sigma_{\mathrm{Ho}}\right)^{2} / e \mu_{\mathrm{VB}} \sigma_{\mathrm{VB}}$.

Fitting to the experimental data made in the frame of this simple model (see Fig. 4) allowed determining activation energies $E_{3}$ as $18 \pm 1 \mathrm{meV}, 19 \pm 1 \mathrm{meV}$, $21 \pm 1 \mathrm{meV}$ for samples Mn I, Mn II, and Mn III, respectively. Taking into account the Mn content determined by GDMS, localization radius of a hole at manganese center, $a_{f}=0.74 \pm 0.1 \mathrm{~nm}$ could be calculated. This value is smaller than hydrogen-like radius, even for heavy holes in InP equal to about $1.2 \mathrm{~nm}$ [5].

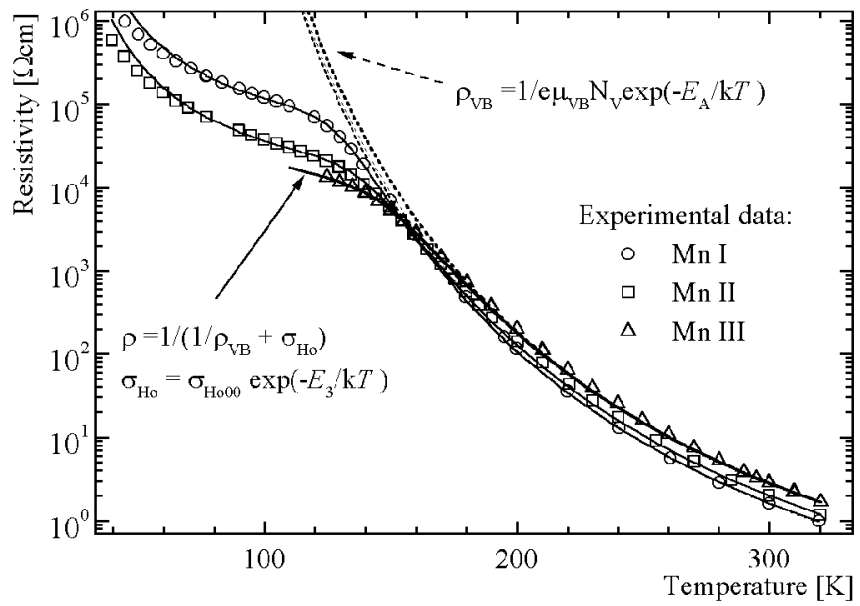

Fig. 4. Temperature dependencies of resistivity for InP:Mn (samples Mn I, Mn II, and Mn III). Experimental points are fitted with solid curves that involve hopping conductivity and with dashed curves that assume hole transport in the valence band only.

The high temperature parts of concentration, mobility and resistivity curves were described by similar parameters for all three samples. It meant that free-hole concentration was similar in all the samples. Its activation energy determined from the fitting was $E_{\mathrm{A}}=(0.205 \pm 0.005) \mathrm{eV}$. 


\section{Conclusions}

Results of the performed experiments, in particular broadening of optical absorption bands, reduction of InP band gap, luminescence shift and especially hopping conductivity observed at low temperatures, suggested formation of Mn-related defect band, in all the investigated samples with Mn concentration of the order of a few times $10^{18} \mathrm{~cm}^{-3}$. For these samples the Fermi level was pinned at $E_{\mathrm{A}}=(0.205 \pm 0.005) \mathrm{eV}$. The width of Mn impurity band was about $20 \mathrm{meV}$ (as estimated from hopping activation energy $E_{3}$ ). Therefore, taking into account low compensation (5\%), one could expect Mn-related level at about $0.22 \mathrm{eV}$ above the top of InP valence band. This value is in agreement with the value reported by Lambert [3]. It is also consistent with observation of Mn-related PL band in the energy range of $0.9-1.2 \mathrm{eV}$. The question arises about the configuration of $\mathrm{Mn}$ center responsible for this defect band. Basing on the results of electron paramagnetic resonance, the center was in $d^{5}$ configuration when occupied by electrons. From hopping parameters the other configuration could be determined as $\operatorname{Mn}\left(d^{5}\right)$ plus a hole of localization radius equal to about $7 \AA$.

The data obtained by time-resolved photoluminescence measurement showed that $\mathrm{Mn}$ defects are fast and efficient recombination centers in InP. The amplitude of luminescence decreased about 500 times in comparison with $\mathrm{InP}$ not doped with $\mathrm{Mn}$. Together, decay times were a few times shorter in the case of $\mathrm{D}-\mathrm{A}$ recombination, and roughly two orders of magnitude shorter in the case of free carriers. The hole recombination coefficient was about $R_{\mathrm{Mn}}=10^{-5} \mathrm{~cm}^{3} / \mathrm{s}$, which allowed to estimate the Mn cross-section for hole capture $\sigma_{p}=2 \times 10^{-12} \mathrm{~cm}^{2}$.

\section{Acknowledgments}

This work was partially supported by the State Committee for Scientific Research, in particular by grants Nos. PBZ-KBN-044/P03/2001 and 115/E-343/ SPUB-M/5.PR UE/DZ 255/2001-2003 and partially by EC under the project FENIKS (G5RD-CT-2001-00535).

\section{References}

[1] K.W. Edmonds, K.Y. Wang, R.P. Campion, A.C. Neumann, N.R.S. Farley, B.L. Gallagher, C.T. Foxon, Appl. Phys. Lett. 81, 4991 (2002).

[2] Yan Dawei, B.C. Cavenett, M.S. Skolnick, J. Phys. C, Solid State Phys. 16, L647 (1983).

[3] B. Lambert, B. Clerjaud, C. Naud, B. Deveaud, G. Picoli, Y. Toudic, in: Proc. 13th Int. Conf. on Defects in Semiconductors, Coronado (USA), Eds. L.C. Kimerling, J.M. Parsey, Jr., Metallurgical Society of AIME, New York 1985, p. 1141.

[4] B.I. Shklovskii, A.L. Efros, Electronic Properties of Doped Semiconductors, Springer-Verlag, Berlin 1984.

[5] Landolt-Börnstein, Numerical Data and Functional Relationships in Science and Technology, New Series, Group III, Vol. 17a, Ed. O. Madelung, Springer Verlag, Berlin 1982, p. 281. 